\title{
A Global Analysis of CYP51 Diversity and Azole Sensitivity in Rhynchosporium commune
}

\author{
Patrick C. Brunner, Tryggvi S. Stefansson, James Fountaine, Veronica Richina, and Bruce A. McDonald
}

\begin{abstract}
First, second, fourth, and fifth authors: Plant Pathology, Institute of Integrative Biology, ETH Zurich, CH-8092 Zurich, Switzerland; and third author: Scotland's Rural College (SRUC), West Mains Road, Edinburgh UK, EH9 3JG. Current address for T. S. Stefansson: Algalif Iceland, Bogatrod 10, 235 Reykjanesbaer, Iceland. Current address for J. Fountaine: Syngenta Jealott's Hill Research Centre, Bracknell, Berkshire UK, RG42 6EY. Accepted for publication 24 November 2015.
\end{abstract}

\begin{abstract}
Brunner, P. C., Stefansson, T. S., Fountaine, J., Richina, V., and McDonald, B. A. 2016. A global analysis of CYP51 diversity and azole sensitivity in Rhynchosporium commune. Phytopathology 106:355-361.

CYP51 encodes the target site of the azole class of fungicides widely used in plant protection. Some ascomycete pathogens carry two CYP51 paralogs called CYP51A and CYP51B. A recent analysis of CYP51 sequences in 14 European isolates of the barley scald pathogen Rhynchosporium commune revealed three CYP51 paralogs, CYP51A, CYP51B, and

We analyzed a global collection of nearly $400 \mathrm{R}$. commune isolates to determine if these findings could be extended beyond Europe. Our results strongly support the hypothesis that $C Y P 51 A$ played a key role in the emergence of azole resistance globally and provide new evidence that the CYP51A gene in $R$. commune has further evolved, presumably in response to azole exposure. We also present evidence for recent longdistance movement of evolved CYP51A alleles, highlighting the risk associated with movement of fungicide resistance alleles among international trading partners.
\end{abstract} a pseudogene called CYP51A-p. The same analysis showed that CYP51A exhibits a presence/absence polymorphism, with lower sensitivity to azole fungicides associated with the presence of a functional CYP51A.

CYP51 encodes the target site of the azole class of fungicides widely used in agriculture and medicine. The genomes of some ascomycete fungi contain two CYP51 paralogs, called CYP51A and $C Y P 51 B$, that both encode the sterol 14 alpha-demethylase function needed to synthesize ergosterol, a vital component of fungal cell membranes. Applications of azole fungicides impose strong selection that favors mutations in promoter or coding regions of either CYP51 gene, leading to the emergence of azole resistance. In the wheat pathogen Zymoseptoria tritici, the CYP51B paralog (annotated as CYP74 in the IPO323 reference genome) has evolved many resistance mutations in both promoter and coding regions as a result of azole selection (Cools and Fraaije 2013, and references therein). In the human pathogen Aspergillus fumigatus, the CYP51A paralog has evolved to provide azole resistance (Camps et al. 2012).

The barley scald pathogen Rhynchosporium commune has been exposed to azole fungicides in the United Kingdom (UK) since 1975 and has evolved a well-documented decrease in azole sensitivity over several decades (Kendall et al. 1993; Zhan et al. 2008). A recent $\mathrm{Q}_{\mathrm{ST}} / \mathrm{F}_{\mathrm{ST}}$ analysis of nine global field populations of $R$. commune revealed significant differences among populations in the level of azole sensitivity (Stefansson et al. 2013), but the genetic basis of these differences was not considered. A recent analysis of CYP51 sequences in $R$. commune by Hawkins et al. (2014) revealed that $R$. commune carries three CYP51 paralogs, called CYP51A, $C Y P 51 B$, and a duplicated copy of CYP51A with frameshift

Corresponding author: B. A. McDonald;

E-mail address: bruce.mcdonald@usys.ethz.ch

*The $\boldsymbol{e}$-Xtra logo stands for "electronic extra" and indicates that five supplementary figures, two supplementary tables, and one supplementary method file are published online.

http://dx.doi.org/10.1094/PHYTO-07-15-0158-R

(C) 2016 The American Phytopathological Society
Additional keywords: neofunctionalization, intragenic recombination, selection.

mutations that created a pseudogene called CYP51A-p. Hawkins et al. (2014) reported the surprising discovery that the $C Y P 51 A$ gene shows a presence/absence polymorphism, with lower sensitivity to azole fungicides associated with the presence of a functional $C Y P 51 A$. They also showed that CYP51A was present at a very low frequency in UK populations of $R$. commune between approximately 1890 and 1985, after which the frequency of CYP51A increased rapidly. In contrast, azole sensitivity in the 1985 survey of UK populations of $R$. commune was 5 - to 10 -fold lower than the baseline sensitivity measured in 1975 to 1981 (Kendall et al. 1993). Based on these findings, Hawkins et al. (2014) proposed that the increase in frequency of CYP51A is responsible for much of the decrease in azole sensitivity in UK populations of $R$. commune.

The findings of Hawkins et al. (2014) included detailed analyses of only $14 R$. commune strains, including 12 isolates coming from UK and one each coming from Finland and France. We set out to determine if the findings reported by Hawkins et al. (2014) were relevant in field populations of $R$. commune originating from other barley-growing regions of the world. We also sought to determine if any of the CYP51 genes have evolved in response to selection due to azole applications. Through our analyses of a global collection of nearly $400 R$. commune isolates, we confirmed that most of the findings reported by Hawkins et al. (2014) are valid worldwide. We support their hypothesis that CYP51A played a key role in the emergence of azole resistance globally and present new evidence that the CYP51A gene has continued to evolve, likely as an adaptive response to azole exposure. We report the accumulation of additional nonsynonymous substitutions as well as evidence of intragenic recombination in $C Y P 51 A$, similar to findings reported in earlier analyses of $C Y P 51 B$ in Z. tritici (Brunner et al. 2008; Estep et al. 2015). We also find evidence for recent long-distance movement of evolved CYP51A alleles among continents, highlighting the risk posed by movement of fungicide resistance alleles among international trading partners. 


\section{MATERIALS AND METHODS}

Origin of isolates. $R$. commune strains were collected as single spore isolations from naturally infected barley fields in 10 countries on four continents and stored on silica gel at $-80^{\circ} \mathrm{C}$ until used for experiments. Most populations included in these analyses were described in previous studies of quantitative traits and population genetics (Stefansson et al. 2013, 2014). In addition to the previously described Swiss population collected in 2003, we included six Swiss populations collected between 1999 and 2001 to enable a more detailed regional analysis of CYP51A frequencies in field populations. UK isolates were collected in Scotland in 2009 and 2013 along transects in untreated spring barley fields planted to cultivar Optic at the SRUC Bush Estate near Edinburgh using the protocols described in McDonald et al. (1999). The same location, cultivar, and methodology were used in both collection years for the UK isolates. Isolates included in these analyses are summarized in Table 1 and Supplementary Table S1.

Fungicide sensitivity assays. The assay to measure fungicide sensitivity was described previously (Stefansson et al. 2014). Briefly, $\sim 100$ spores of each isolate were spread across potato dextrose agar petri plates amended with either $0.025 \mathrm{ppm}$ or $0.1 \mathrm{ppm}$ of the azole fungicide cyproconazole. Isolates were randomized and plates were incubated at $18^{\circ} \mathrm{C}$ in the dark. Resulting single-spore fungal colonies were photographed through the petri dish lid four times over a 12-day period using a digital camera under standardized conditions. The colony diameter (in millimeters) was measured with the image analysis software APS Assess 2.0 (Lamari 2002). Fungicide resistance was measured by dividing the colony growth rate in the presence of fungicide by the colony growth rate in the absence of fungicide. The number of isolates screened for fungicide resistance in each population is given in Table 1.

Multiplex PCR to determine the presence/absence polymorphism of CYP51A and CYP51A-p. All PCR primers were designed with the program Primer 3 (Untergasser et al. 2012) using published gene sequences (GenBank accessions KF753678-80 for $C Y P 51 B$, KF753687 for $C Y P 51 A$, and KF753684-86 for $C Y P 51 A-p$ ). The functional $C Y P 51 A$ gene was distinguished from the closely related CYP51A-p using a multiplex PCR approach. Primer A (RcCYP51_2892_F) was the conserved forward primer for both genes. Primer B (RcCYP51_3266_R-f) was the specific reverse primer for the functional $C Y P 51 A$ allele, resulting in a PCR amplicon of $400 \mathrm{bp}$. Primer C (RcCyp51_3382_R-p) was the specific reverse primer for the CYP51A- $p$ pseudogene, resulting in a PCR amplicon of $500 \mathrm{bp}$. Details of the multiplex PCR reaction and primer sequences are given in the Supplementary Method File S1.
Sequencing of CYP51A, CYP51A-p, and CYP51B alleles. Entire $C Y P 51$ genes were sequenced using standard protocols as previously described (Brunner et al. 2008). Briefly, genes were first PCR-amplified using primer pairs that flanked the entire coding region. PCR products were purified and sequenced using the BigDye Terminator v3.0 Cycle Sequencing kit (Applied Biosystems, Foster City, CA) according to the manufacturer's instructions on an ABI PRISM 3130xl Genetic Analyzer. Because of the length of the genes $(\sim 1,700 \mathrm{bp})$, primers used for sequencing were the gene-flanking PCR primers with additional internal primers to produce overlapping sequences. Obtained nucleotide sequences of CYP51B and CYP51A were aligned on the level of translated proteins using TranslatorX (Abascal et al. 2010) and the implemented MAFFT algorithm (Katoh et al. 2009), and edited using BIOEDIT (Hall 1999).

Sequence analyses. DnaSP v5 (Librado and Rozas 2009) was used to calculate summary statistics of molecular diversity. Rarefaction was used to correct numbers of haplotypes for unequal sample sizes. Rarefaction analysis followed the method described by Simberloff (1978) as implemented in an online calculator (http://www.biology.ualberta.ca/jbrzusto/rarefact.php\#Inputs). Parsimony networks showing the relationships among haplotypes were constructed using the program TCS 1.21 (Clement et al. 2000).

Recombination can play an important role in generating allelic diversity. To infer the contribution of intragenic recombination to the development of resistant Cyp51A isoforms, we assumed that the total observed genetic diversity is composed of variation due to mutations $(M)$ and variation due to intragenic recombination $(R)$. The genealogical relationships among haplotypes and the dynamics of sequence evolution can be modeled using the coalescent process. The coalescent simulations implemented in DnaSP are based on a neutral infinite-sites model and assume a large constant population size. We addressed the potential pitfall of assessing genes under selection by analyzing only synonymous nucleotide sites. Furthermore, we tested all three genes individually and also in a concatenated data set for constant population size using the program BEAST by comparing the likelihoods of different population models applied to the data set (Drummond et al. 2012). In all comparisons, the constant-population-model had likelihoods $\sim 16$ to 300 times greater than the population growth models, justifying the use of DnaSP.

DnaSP was applied to our data sets to simulate the expected number of haplotypes based strictly on the coalescent process using the option of no intragenic recombination $(R=0)$. The resulting number of simulated nonrecombinant haplotypes $\left(H_{M}\right)$ was compared with the total number of observed haplotypes to infer the proportion of haplotypes generated by recombination $\left(H_{R}\right)$. Different kinds and strengths of selection may affect the inference of recombination, making a comparison between the CYP51A

TABLE 1. Origin, year of collection and sample sizes $(N)$ of Rhynchosporium commune populations included in analyses of $C Y P 51$ sequences, $C Y P 51 A$ presenceabsence polymorphism, and fungicide sensitivity ${ }^{\mathrm{a}}$

\begin{tabular}{|c|c|c|c|c|c|c|c|c|}
\hline \multirow[b]{2}{*}{ Origin } & \multirow[b]{2}{*}{ Abbreviation } & \multirow[b]{2}{*}{ Year } & \multicolumn{3}{|c|}{ Sequencing $(N)$} & \multicolumn{2}{|c|}{ CYP51A detection } & \multirow[b]{2}{*}{ Fungicide assay ${ }^{b}$} \\
\hline & & & CYP51B & CYP51A & $C Y P 51 A-p$ & $N$ tested & CYP51A \% & \\
\hline Ethiopia & ETH & 2003 & 8 & - & 16 & 14 & 0 & $13 / 0$ \\
\hline United Kingdom & UK & 2009-2013 & 27 & 29 & 8 & 72 & $100 \%$ & $0 / 22$ \\
\hline Finland & FIN & 2000 & 8 & - & 12 & 14 & 0 & $14 / 0$ \\
\hline Iceland & ICE & 2008 & 8 & - & - & 14 & 0 & $12 / 0$ \\
\hline Norway & NOR & 2000 & 8 & - & 4 & 14 & 0 & $14 / 0$ \\
\hline Spain & SPN & 2005 & 4 & 2 & 4 & 10 & $20 \%$ & $10 / 1$ \\
\hline Switzerland & SWI & 1999-2003 & 8 & 16 & 8 & 220 & $76 \%$ & $8 / 7$ \\
\hline California, USA & USA & 1989 & 8 & - & 8 & 13 & 0 & $14 / 0$ \\
\hline New Zealand & NZL & 2009 & 8 & 45 & 4 & 13 & $85 \%$ & $3 / 34$ \\
\hline Australia & AUS & 1999 & 5 & - & - & 13 & 0 & $12 / 0$ \\
\hline Syria & SYR & 2003 & 6 & - & - & & & $0 / 0$ \\
\hline Total/average & & & 92 & 92 & 64 & 397 & 28 & $100 / 64$ \\
\hline
\end{tabular}

a More information on different sampling sites or years used to calculate CYP51A frequencies are given in Supplementary Table S1.

b The first number is the count of assessed isolates without the CYP51A gene. The second number is the count of assessed isolates with the CYP51A gene. 
isoforms unreliable. To avoid this potential problem, final recombination analyses were based solely on synonymous sites. Conducting the same analyses using all nucleotide sites yielded results in agreement with the reduced data set (Supplementary Fig. S1).

Selection analyses were conducted using different approaches. DnaSP was used to estimate allele frequency deviations from neutral expectations based on Tajima's $D$ (Tajima 1989) for all three paralogs. Codon-based approaches were applied to assess the ratio of synonymous to nonsynonymous substitutions ( $\mathrm{dN} / \mathrm{dS}$, also denoted as $\omega$ ). The Z-test implemented in MEGA (Tamura et al. 2013) compares pairwise estimates of $\mathrm{dN} / \mathrm{dS}$ to test the hypotheses of neutrality $(\mathrm{dN}=\mathrm{dS})$, diversifying selection $(\mathrm{dN}>\mathrm{dS})$, and purifying selection $(\mathrm{dN}<\mathrm{dS})$. Finally, we applied the phylogenybased maximum likelihood approach of the PAML program package (Yang 2007). We were specifically interested to compare signatures of selection that occurred after the gene duplication that led to CYP51B and CYP51A by applying the implemented "branch model" tests (Supplementary Fig. S2). Since deletion mutations caused reading frame shifts in the $C Y P 51 A$-pseudogene, these codon-based approaches could only be carried out for $C Y P 51 B$ and $C Y P 51 A$.

\section{RESULTS}

Presence/absence of the CYP51A gene and its association with azole sensitivity. A total of 397 isolates of $R$. commune from 18 global field populations were tested for the presence or absence of CYP51A. Table 1 summarizes the presence/absence polymorphism for each sampling site. CYP51A was found only in $R$. commune isolates sampled from barley fields in the UK (100\% of isolates), New Zealand ( $\sim 85 \%)$, Switzerland ( 76\%), and Spain $(\sim 20 \%)$. Average azole sensitivity was significantly lower in strains carrying the CYP51A gene compared with isolates lacking the gene (Fig. 1). Mean relative growth rates on $0.025 \mathrm{ppm}$ of cyproconazole were 0.94 and $0.43(t=-14.3, P<0.001)$, and 0.89 and 0.01 on $0.1 \mathrm{ppm}$ of cyproconazole $(t=-38.6, P<0.001)$ for strains with or without $C Y P 51 A$, respectively. A screen of 220 $R$. commune isolates sampled between 1999 to 2001 from six barley fields distributed across Switzerland found that the frequency of isolates carrying CYP51A ranged from 45 to $100 \%$ among fields, with a country-wide average of $76 \%$.

Sequence analyses of CYP51B, CYP51A-p, and CYP51A. The entire $C Y P 51 B$ gene was sequenced in 92 global isolates representing all sampled regions. A total of six sequence variants encoding four amino acid isoforms were identified. Only the most common amino acid isoform was found in all populations except for the UK population that had all four isoforms (Fig. 2A). CYP51B had the lowest nucleotide diversity $\left(\mathrm{p}_{\mathrm{i}}=0.009\right)$ and the lowest haplotype diversity $\left(\mathrm{H}_{\mathrm{d}}=0.48\right)$ among the three genes (Table 2$)$. Average nucleotide sequence similarity compared with the CYP51A gene was $57 \%$, and amino acid similarity was similarly low with a mean of $53 \%$. No correlation was found between the degree of azole sensitivity and variation in the $C Y P 51 B$ gene.

The entire CYP51A-p gene was sequenced in 64 global isolates including 8 of the 11 sampled regions. Nucleotide diversity was $2.7 \times$ higher than found for $C Y P 51 B$, with $\mathrm{p}_{\mathrm{i}}=0.0024$. A total of 16 nucleotide haplotypes were found $\left(\mathrm{H}_{\mathrm{d}}=0.82\right)$. The most common haplotype was distributed globally, with the largest number of haplotypes (8) found in Finland and the smallest number (1) found in Ethiopia (Fig. 2B). Alignment of CYP51A- $p$ and CYP51A sequences was straightforward because their mean nucleotide sequence similarity was $93 \%$. The most prominent differences were a four base-pair deletion ( $\Delta 419$ to 422$)$ that was found in all isolates and a one base pair deletion $(\Delta 1622)$ that was shared among ca. $50 \%$ of the isolates. Both deletions caused shifts in the reading frame, resulting in numerous premature stop codons, a common observation in pseudogenes.
The entire CYP51A gene was sequenced in 92 isolates from Spain (2), New Zealand (45), UK (29), and Switzerland (16), differentiating 24 sequence haplotypes. Rarefaction analysis showed that the UK population had the highest sequence diversity, followed by the Swiss and New Zealand populations. Compared with the CYP51A-p pseudogene, there were 50\% fewer segregating sites, but slightly more haplotypes for the functional CYP51A following rarefaction (Table 2; Supplementary Fig. S3). Figure 2C shows the haplotype network summarizing the geographical distribution and genetic relationships among the CYP51A alleles. Each field population had more than one CYP51A haplotype and three haplotypes were shared among populations. The UK and Swiss populations carried 11 and 7 private haplotypes, respectively. There was little evidence for geographical differentiation among populations; three of the four CYP51A haplotypes found in New Zealand were also present in UK and Switzerland. But there were many reticulations consistent with intragenic recombination in the CYP51A haplotype network (Fig. 2C). The haplotype alignments of all three genes are provided in Supplementary Table S2.

The amount of intragenic recombination was explored further using additional analyses based on the coalescent. Figure 3A shows that only $C Y P 51 A$ had a significantly lower number of simulated nonrecombinant haplotypes compared with observed haplotypes, suggesting a high proportion of the existing CYP51A haplotypes were generated through intragenic recombination. The normalized proportions of recombinant haplotypes $\left(H_{R}\right)$ and mutation haplotypes $\left(H_{M}\right)$ is given in Figure $3 \mathrm{~B}$. Consistent with the amount of reticulation observed in the network analyses (Fig. 2), no intragenic recombinants were inferred for $C Y P 51 B$, but the $C Y P 51 A-p$ pseudogene had an $H_{R}$ fraction of $8 \%$ while the functional $C Y P 51 A$ gene had an $H_{R}$ fraction of $35 \%$. More detailed analyses of the CYP51A data revealed that the UK subpopulation had the highest proportion of recombinant haplotypes $(61 \%)$ followed by the Swiss (40\%) and New Zealand subpopulations (17\%) (Supplementary Fig. S4).

Selection analyses. The site frequency spectrum (SFS) of DNA sequences is shaped by selective forces and/or demographic processes such as population expansions or bottlenecks. Demographic processes are expected to equally influence the SFS of all genes in a population while selective forces operate only on genes under selection. Diversity patterns of the SFS were summarized using
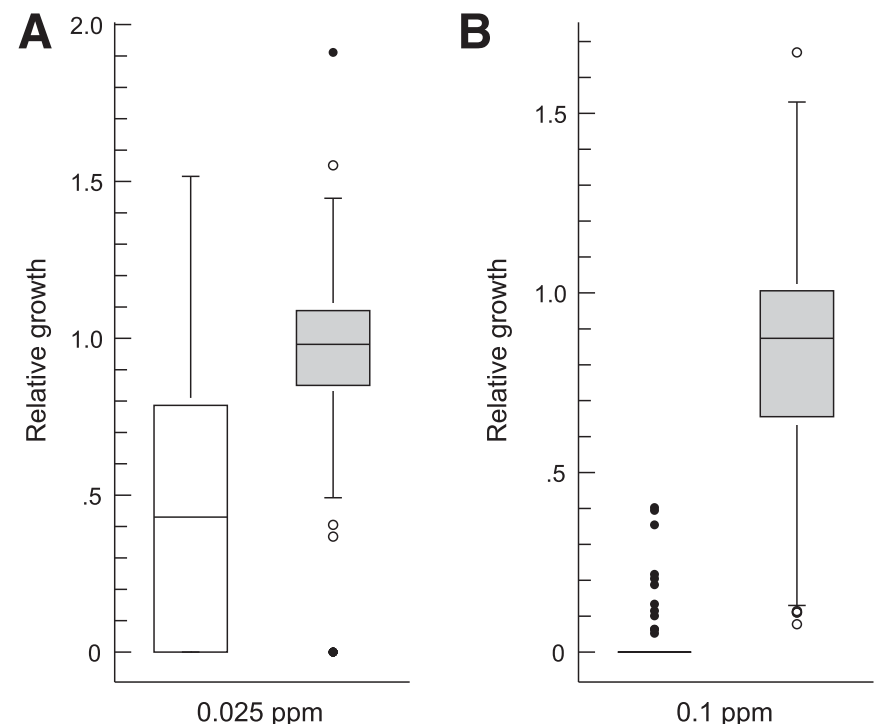

$0.1 \mathrm{ppm}$

Fig. 1. In vitro fungicide sensitivity assays using cyproconazole concentrations of $\mathbf{A}, 0.025 \mathrm{ppm}$ and $\mathbf{B}, 0.10 \mathrm{ppm}$. White box-plots show the relative growth rates of Rhynchosporium commune isolates without the CYP51A gene. Gray box-plots show the relative growth rates of isolates with the CYP51A gene. 
A
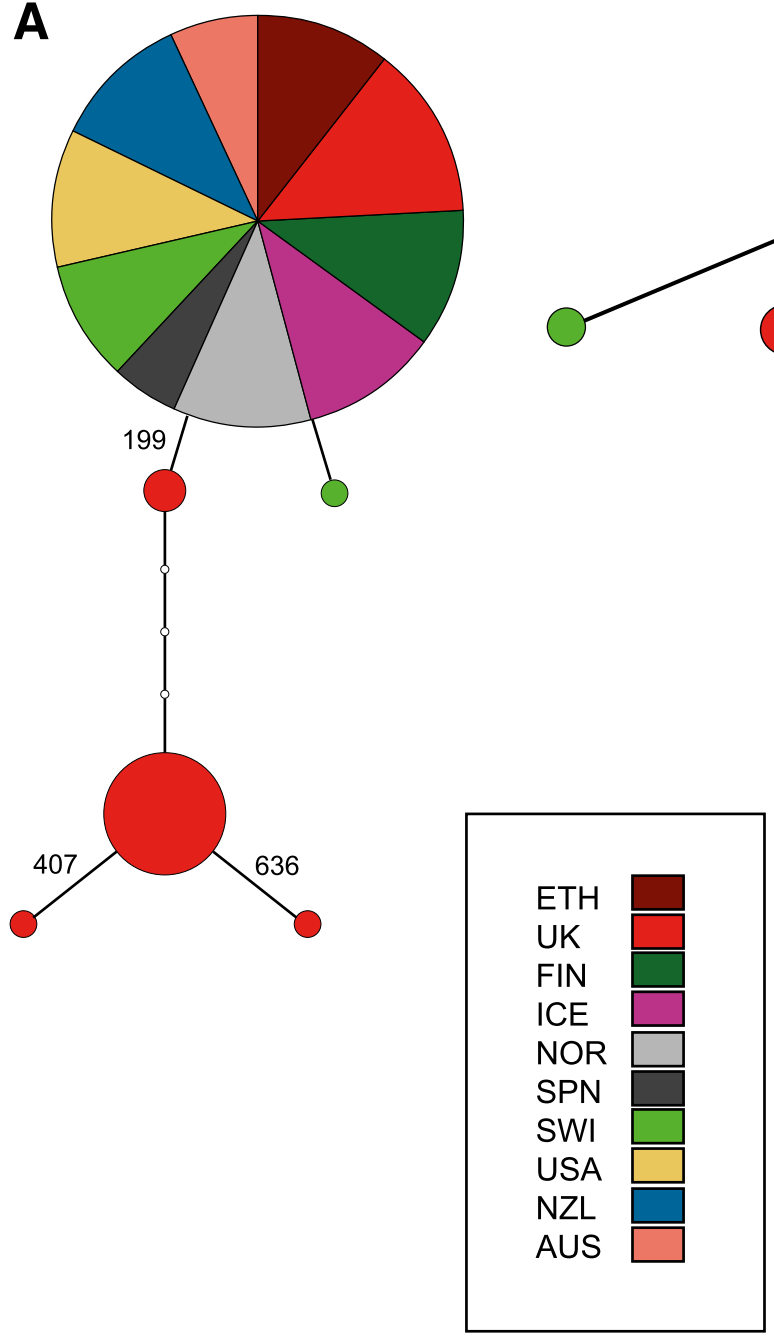

B

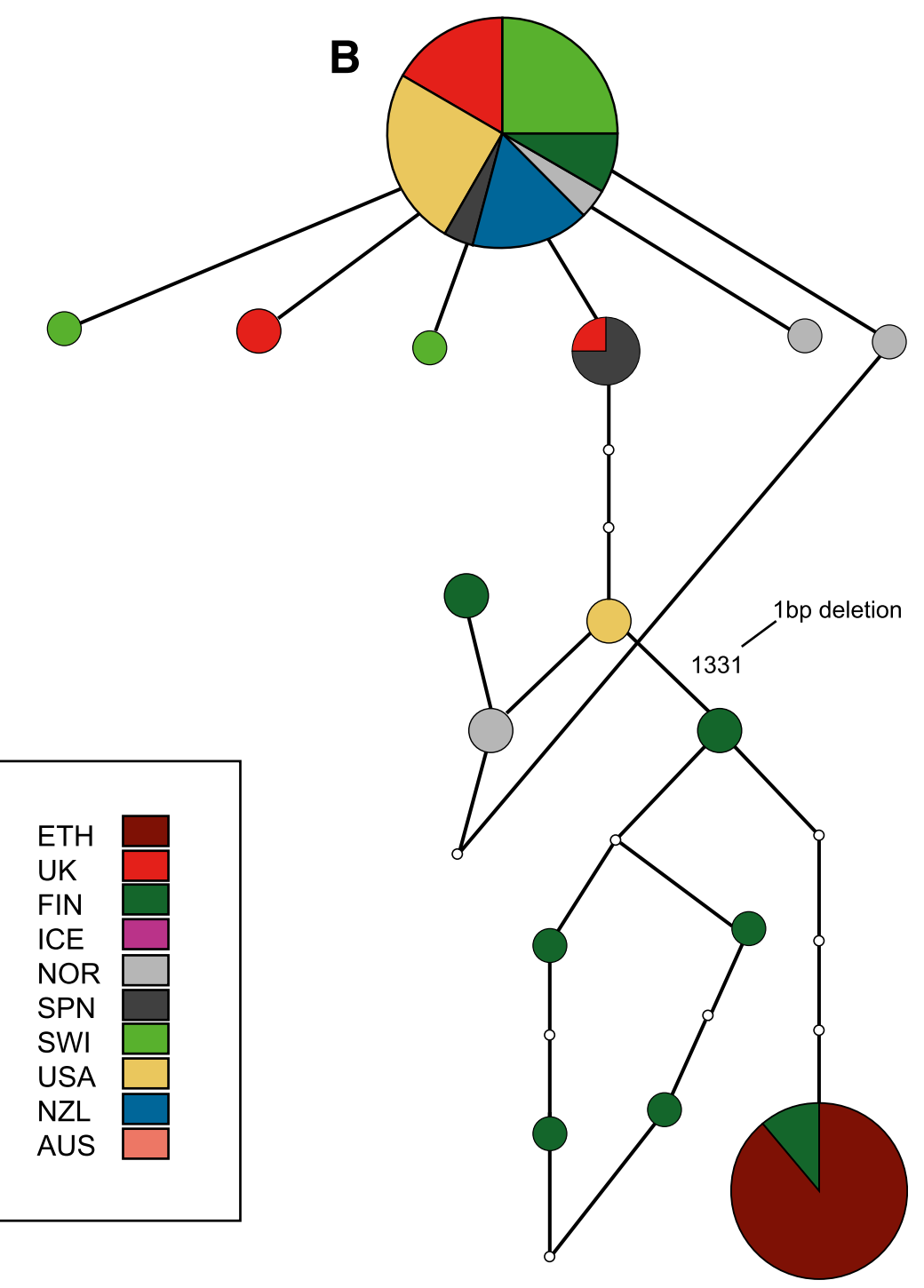

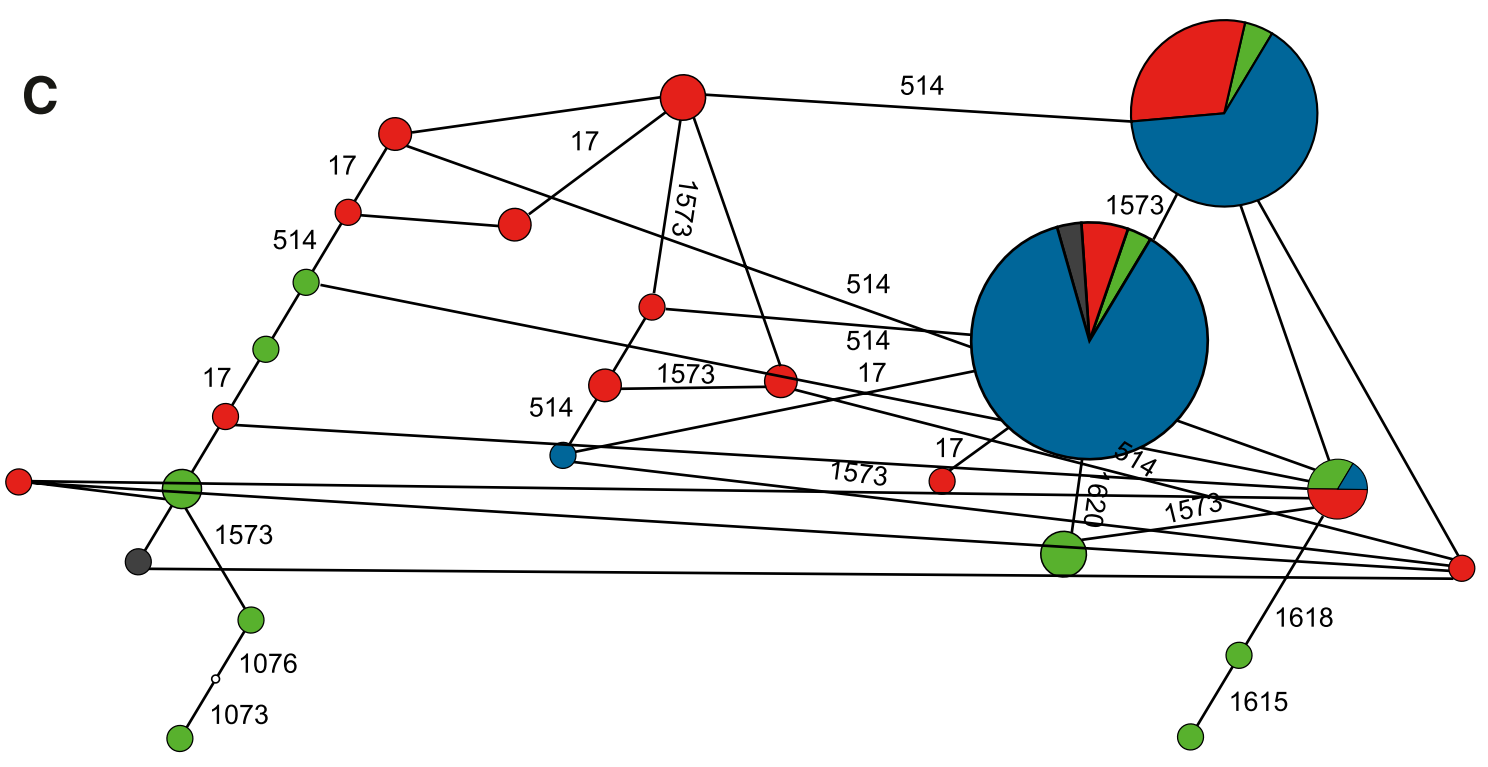

Fig. 2. Haplotype networks for each CYP51 gene. Ellipse sizes reflect the frequency of each haplotype and colors correspond to the origin of the Rhynchosporium commune isolates using abbreviations given in Table 1. Small open circles represent missing haplotypes in the network. Each connecting branch represents a single nucleotide mutation. A, Haplotype network for the $C Y P 51 B$ gene. Numbers on branches indicate nonsynonymous nucleotide changes with respect to the $C Y P 51 B$ transcript sequence of $R$. commune isolate UK7 (GB-accession 665823869). B, Haplotype network for the CYP51A-p pseudogene. Numbers on branches indicate nucleotide changes with respect to the CYP51A transcript sequence of $R$. commune isolate 13-13 that result in reading frame shifts. C, Haplotype network for the CYP51A gene. Numbers on branches indicate nonsynonymous nucleotide changes with respect to the CYP51A transcript sequence of $R$. commune isolate 13-13 (GB-accession 665823856). Note that this network shows a strong pattern of reticulation; i.e., many haplotypes are connected with more than one haplotype because the same mutations appear in different parts of the network, consistent with intragenic recombination. 
Tajima's $D$ statistic (Table 2). We observed contrasting values of Tajima's $D$ among the three genes, indicative of different selective forces operating on these genes. Supporting the hypothesis that $C Y P 51 A-p$ is a pseudogene, we found that Tajima's $D$ for this gene is virtually zero $(D=0.06)$, as expected for a neutral gene. In contrast, $C Y P 51 B$ has a positive value $(D=0.44)$, while $C Y P 51 A$ has a negative value $(D=-0.31)$ suggesting that different types of selection were acting on these genes, though none of these values were significant.

Results of selection analyses based on synonymous and nonsynonymous substitutions are summarized in Table 3 . On the codon level there was a fivefold excess of nonsynonymous compared with synonymous substitutions in $C Y P 51 A$, leading to a significantly higher $\omega$ than observed for the CYP51B gene $(t$ test; $P<0.001)$. Under neutrality, $d N$ equals $\mathrm{dS}$, hence $\omega=\mathrm{dN} / \mathrm{dS}=1$. The Z-test confirmed that the $\omega$ of $C Y P 51 B$ was significantly lower than expected under neutrality, suggesting that $C Y P 51 B$ is under significant purifying selection $(\mathrm{dN}<\mathrm{dS})$. However, the Z-test did not reject the null hypothesis of neutrality $(\mathrm{dN}=\mathrm{dS}, P=0.35)$ for CYP51A.

The PAML analyses did not infer $\omega>1$ for the phylogenetic branch leading to the $C Y P 51 B$ haplotypes $(\omega 1=0.423)$. Furthermore, the likelihood of the two-ratio selection model was not significantly different from the one-ratio model $(P=0.157)$. This supports the previous analyses indicating that $C Y P 51 B$ is under strong purifying selection. In contrast, PAML inferred a significantly positive $\omega 1=$ $2.026(P<0.001)$ for the branch leading to $C Y P 51 A$. Does this finding reflect a real signature of diversifying selection leading to CYP51A or just a relaxation of purifying selection pressure following the gene duplication? To answer this question, we contrasted this result against a model of neutrality by fixing $\omega 1=1$, which showed that the positive selection model performed significantly better $(P=0.045)$, suggesting that diversifying selection operated during the evolution of CYP51A.

\section{DISCUSSION}

Our analyses of a global collection of $R$. commune strains largely confirms the findings Hawkins et al. (2014) described for UK isolates and extends their findings to a global scale. The $C Y P 51 B$ gene was found in all isolates globally, but there was little diversity for DNA or amino acid sequences and no association was found between $C Y P 51 B$ haplotypes and azole sensitivity. The CYP51A-p pseudogene was found in all $R$. commune isolates globally, but also was not correlated with azole sensitivity. In stark contrast, CYP51A showed a presence/absence polymorphism that was significantly correlated with azole sensitivity globally. In addition, we found evidence that positive diversifying selection is operating on $C Y P 51 \mathrm{~A}$, likely reflecting selection for resistance to azoles. The sharing of CYP51A and CYP51A-p alleles among field populations on different continents is consistent with recent gene flow facilitating exchange of these alleles among global populations.

Our selection analyses and the haplotype network associated with $C Y P 51 B$ indicate that this gene exhibits purifying selection and likely operates under functional constraints, though it is noteworthy that only the UK population carried all four protein isoforms.

TABLE 2. Summary statistics of DNA sequence diversity found for three CYP51 paralogs in global collections of Rhynchosporium commune ${ }^{\mathrm{a}}$

\begin{tabular}{lcccrrrrrr}
\hline & $N$ & Ex & In & \multicolumn{1}{c}{$\mathrm{S}$} & $\mathrm{h}$ & $\mathrm{h}_{\mathrm{R}}$ & $\mathrm{Hd}$ & $\pi$ & Tajima's $D$ \\
\hline CYP51B & 92 & 1,581 & 128 & 8 & 6 & 6 & 0.480 & 0.0009 & 0.44 \\
CYP51A- $p$ & 64 & 1,619 & 292 & 21 & 16 & 16 & 0.820 & 0.0024 & 0.06 \\
CYP51A & 92 & 1,623 & 292 & 10 & 24 & 18 & 0.808 & 0.0012 & -0.31 \\
\hline
\end{tabular}

a $N$ is number of isolates sequenced. Ex is length of exon region in base pairs. In is length of intron region in base pairs. $\mathrm{S}$ is number of segregating (polymorphic) sites. $\mathrm{h}$ is number of distinct haplotypes. $\mathrm{h}_{\mathrm{R}}$ is number of haplotypes after rarefaction $(N=64)$. Hd is haplotype diversity. $\pi$ is nucleotide diversity.
The $C Y P 51 B$ gene in the Dothidiomycete Zymoseptoria tritici plays an important role in azole resistance and has evolved considerably in response to azole applications, especially in Europe (Cools and Fraaije 2013, and references therein), but Z. tritici does not appear to carry a $C Y P 51 A$ homolog. In other fungi that carry both $C Y P 51 A$ and $C Y P 51 B$, it appears more common to develop mutations in CYP51A and/or to overexpress this paralog (e.g., in Aspergillus fumigatus and Penicillium digitatum) in response to azole selection pressure. The presence of CYP51A was shown to reduce intrinsic azole sensitivity in both Fusarium graminearum (Fan et al. 2013; Liu et al. 2011) and Magnaporthe grisea (Yan et al. 2011).

One of the interesting discoveries in the $R$. commune genome sequence was the presence of a CYP51A pseudogene (Hawkins et al. 2014). We confirmed that the CYP51A-p gene is distributed globally and carries a high amount of diversity as expected for a nonfunctional and neutrally evolving pseudogene. As reported by Hawkins et al. (2014), we did not correlate CYP51A-p diversity with azole sensitivity.

The functional CYP51A gene was found only in the UK, Switzerland, Spain, and New Zealand. This distribution pattern supports the hypothesis that the frequency of CYP51A has increased mainly in regions where azole fungicides have been applied regularly. The finding also supports our earlier analyses of azole sensitivity showing that field populations in Switzerland and New Zealand were more resistant to azoles than seven other global

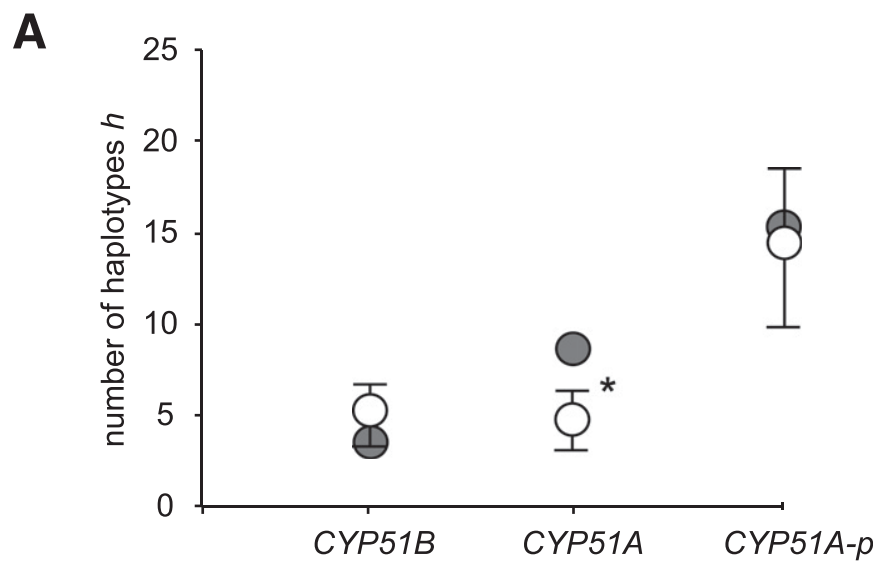

B

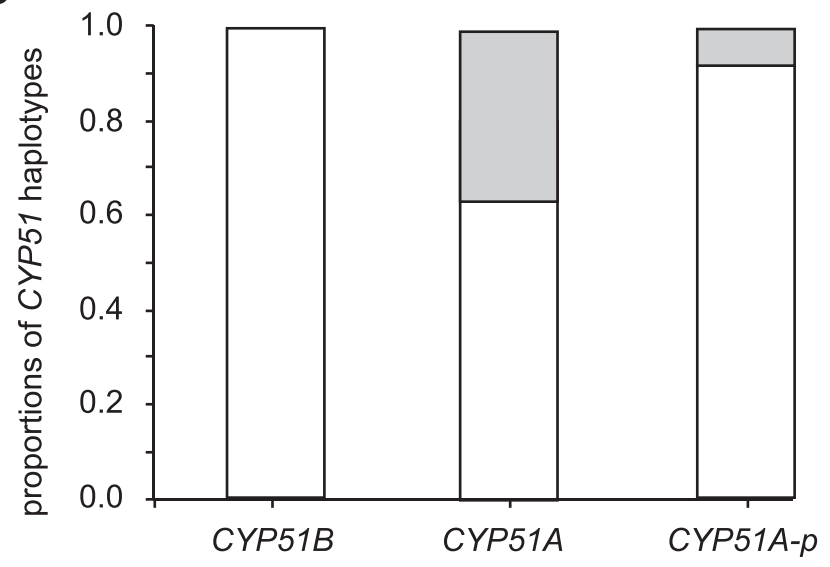

Fig. 3. Simulation analyses of recombinant and nonrecombinant haplotypes using the coalescent approach implemented in DnaSP. A, Observed numbers of haplotypes for all three CYP51 genes are indicated in gray. The simulated numbers of nonrecombinant haplotypes together with their $95 \%$ confidence intervals are indicated in white. Asterisks indicate significant differences between simulated and observed numbers of haplotypes. B, The normalized proportions of simulated nonrecombinant haplotypes (white bars) and haplotypes derived by mutations (gray bars) for each CYP51 gene. 
populations (Stefansson et al. 2014). We did not detect CYP51A in Ethiopia, Australia, United States, Iceland, or Norway, but only one barley field from each country was included in the analysis and sample sizes were small, so it is possible that all of these populations carry the CYP51A gene at a low frequency. It is also possible that selection due to azole use has not been sufficiently high or sufficiently long in these areas to increase the frequencies of strains carrying the CYP51A gene. For example, a 10-year difference in sampling time could explain the detection of the CYP51A gene in New Zealand but not in Australia. Hawkins et al. (2014) documented a rapid increase in CYP51A starting in 1985 in the UK. The high frequency in three different Swiss barley fields sampled in 1999 suggests that CYP51A increased in frequency in mainland Europe before 1999.

Evidence for selection operating on CYP51A. Hawkins et al. (2014) showed that the CYP51A gene is upregulated $\sim 100$ fold in the presence of azoles and ascribed the observed resistance mainly to this phenomenon. They also found two nonsynonymous substitutions among the 13 CYP51A gene sequences used for their analyses (GenBank accession numbers KF753639 to KF753674) but did not find a correlation between different alleles and azole sensitivity. We found 24 CYP51A alleles among 92 sequenced strains $\left(\mathrm{H}_{\mathrm{d}}=0.808\right)$ and we consider it notable that more alleles were found for the functional CYP51A gene than for the CYP51A pseudogene, even after rarefaction.

Gene duplications are generally believed to be the main source of new genes (Ohno 1970; reviewed by Zhang 2003). Population genetic theories predict that functional divergence between duplicated genes is usually required for their long-term retention. This "neofunctionalization" (NF) hypothesis proposes that after duplication one daughter gene retains the ancestral function while the other copy can gain novel functions (Ohno 1970). On the other hand, deleterious mutations can accumulate in redundant copies and render the gene nonfunctional, (i.e., "pseudogenization"). The CYP51 paralogs in $R$. commune likely emerged through two gene duplication events (Hawkins et al. 2014; Supplementary Fig. S5). The more ancient duplication gave rise to the functional $C Y P 51 B$ and $C Y P 51 A$ genes, while the more recent event duplicated the $C Y P 51 A$ gene and gave rise to the CYP51A-p pseudogene. Hawkins et al. (2014) concluded that $C Y P 51 A$ in $R$. commune demonstrates functional divergence from $C Y P 51 B$ through different patterns of transcriptional regulation, including greater up-regulation in response to azoles. Our genetic analyses indicated that $C Y P 51 A$ and $C Y P 51 B$ are under different selection regimes. Since the duplication event, $C Y P 51 B$ was under strong purifying selection, likely favoring functional optimization. $C Y P 51 B$ can therefore be regarded as the conserved functional CYP51 gene in $R$. commune. On the other hand, CYP51A shows evidence for diversifying selection following gene duplication and exhibits a presence/absence polymorphism. These contrasting patterns of selection are consistent with the theory of NF and support the conclusion of Hawkins et al. (2014). Also in accordance with these predictions is the fate of the CYP51A- $p$ gene that did not functionally diverge from the $C Y P 51 A$ gene, but accumulated deleterious mutations (i.e., the $4 \mathrm{bp}$ and the $1 \mathrm{bp}$ deletions) that rendered the gene nonfunctional. Additional support for our hypothesis of accelerated evolution in the CYP51A gene comes from our analyses of recombination.

Evidence for intragenic recombination in $C Y P 51 A$. Intragenic recombination in a $C Y P 51$ gene can be an important source of genetic variation that may accelerate evolution of fungicide resistance (Brunner et al. 2008). We found evidence for different degrees of intragenic recombination in the $C Y P 51$ genes of $R$. commune. We did not detect any signatures of recombination in $C Y P 51 B$. This is consistent with our previous interpretation that this member of the CYP51 gene family is under strong purifying selection because of its functional importance. We found relatively few recombinant haplotypes in CYP51A-p as evidenced by the low degree of reticulation in the haplotype network and an inferred proportion of only $8 \%$ recombinant haplotypes in our coalescent analyses. In sharp contrast, the functional CYP51A gene has eight times more inferred recombinant haplotypes and exhibits strong reticulation in its haplotype network. This is particularly noteworthy because the two genes are the same size and CYP51A-p is a neutral pseudogene. This suggests that many more of the variants generated by intragenic recombination were retained in $C Y P 51 A$ compared with $C Y P 51 A-p$, indicative of diversifying selection operating on $C Y P 51 A$. The lowest number of $C Y P 51 A$ recombinants was found in New Zealand. The largest number of recombinants was found in UK. Switzerland was intermediate in recombination. These observations support the hypothesis that intragenic recombinants are most favored in environments with the strongest selection for azole resistance. Taken together, we interpret these findings as strong support for the hypothesis that selection due to azoles is driving changes in the Cyp51A amino acid sequence, though this hypothesis will require further functional testing.

Evidence for CYP51 gene flow among populations. Gene flow over long distances (particularly from Europe to Australia and New Zealand) was already reported for $R$. commune based on neutral SSR markers (Linde et al. 2009). The mechanism of movement was proposed to be trade of infected barley seeds (McDonald 2015). Consistent with the earlier findings, we found evidence for gene flow affecting all three CYP51 genes. The much higher genetic diversity of $C Y P 51 A$ in the UK and the shared haplotypes between regions supports the hypothesis of gene flow from UK to Switzerland, Spain, and New Zealand. The low diversity found in New Zealand may reflect a recent introduction of $C Y P 51 A$ alleles from the UK, representing a founder effect. All CYP51A- $p$ haplotypes shared the same 4 bp deletion, consistent with a common origin for this apparently neutral pseudogene. The shared 1 bp deletion observed in several CYP51A- $p$ haplotypes in Finland and Ethiopia (Fig. 2C) also is consistent with gene flow. Taken together, these data illustrate how gene flow may play an important role in moving fungicide resistance alleles that emerge in one region as a result of high levels of fungicide usage to trading partners that may experience a more rapid emergence of resistance when they use the same fungicides (McDonald 2015).

\begin{tabular}{|c|c|c|c|c|c|c|c|c|c|c|c|c|}
\hline & \multirow[b]{3}{*}{$\mathrm{P}_{\mathrm{S}}$} & \multirow[b]{3}{*}{$\mathrm{P}_{\mathrm{N}}$} & \multirow[b]{3}{*}{$\omega$} & \multirow{2}{*}{\multicolumn{3}{|c|}{ Z-test }} & \multicolumn{6}{|c|}{ PAML branch model } \\
\hline & & & & & & & \multicolumn{3}{|c|}{$\omega 1$ selection } & \multicolumn{3}{|c|}{$\omega 1$ neutral } \\
\hline & & & & $\mathrm{dN}=\mathrm{dS}$ & $\mathrm{dN}>\mathrm{dS}$ & $\mathrm{dN}<\mathrm{dS}$ & $\omega 0$ & $\omega 1$ & $-\ln L^{b}$ & $\omega 0$ & $\omega 1$ & $-\ln L^{c}$ \\
\hline CYP51A & 2 & 10 & 0.472 & 0.350 & 1.000 & 0.203 & 0.328 & 2.026 & $10540 *$ & 0.341 & 1.000 & $10542 *$ \\
\hline
\end{tabular}

a Asterisks indicate models that are significantly different from the one-ratio model $\left(-\mathrm{InL}^{\mathrm{d}}\right)$, or models that are significantly different from the neutral model

$\left(-\mathrm{InL}^{\mathrm{c}}\right)$. $\mathrm{P}_{\mathrm{S}}$ is number of synonymous nucleotide changes. $\mathrm{P}_{\mathrm{N}}$ is number of nonsynonymous nucleotide changes. dN is nonsynonymous mutations per nonsynonymous site. $\mathrm{dS}$ is synonymous mutations per synonymous site. $\omega=\mathrm{dN} / \mathrm{dS}$.

b Likelihood ratio test (LRT) compared - $\mathrm{lnL}$ value of this "two-ratio" model against the one-ratio model M0, $-\mathrm{InL}^{\mathrm{d}}=10556$

c LRT compared -lnL value of this neutral model against the selection model. 


\section{ACKNOWLEDGMENTS}

This research was supported by a grant from ETH Zurich (TH-02 07-1). DNA sequence data were collected in the Genetic Diversity Centre of ETH Zurich.

\section{LITERATURE CITED}

Abascal, F., Zardoya, R., and Telford, M. J. 2010. TranslatorX: Multiple alignment of nucleotide sequences guided by amino acid translations. Nucleic Acids Res. 38:W7-W13.

Brunner, P. C., Stefanato, F. L., and McDonald, B. A. 2008. Evolution of the CYP51 gene in Mycosphaerella graminicola: Evidence for intragenic recombination and selective replacement. Mol. Plant Pathol. 9:305-316.

Camps, S. M., Rijs, A. J., Klaassen, C. H., Meis, J. F., O'Gorman, C. M., Dyer, P. S., Melchers, W. J. G., and Verweij, P. E. 2012. Molecular epidemiology of Aspergillus fumigatus isolates harboring the TR34/L98H azole resistance mechanism. J. Clin. Microbiol. 50:2674-2680.

Clement, M., Posada, D., and Crandall, K. A. 2000. TCS: A computer program to estimate gene genealogies. Mol. Ecol. 9:1657-1659.

Cools, H. J., and Fraaije, B. A. 2013. Update on mechanisms of azole resistance in Mycosphaerella graminicola and implications for future control. Pest Manag. Sci. 69:150-155.

Drummond, A. J., Suchard, M. A., Xie, D., and Rambaut, A. 2012. Bayesian phylogenetics with BEAUti and the BEAST 1.7. Mol. Biol. Evol. 29: 1969-1973.

Estep, L. K., Torriani, S. S. F., Zala, M., Anderson, N. P., Flowers, M. D., McDonald, B. A., Mundt, C. C., and Brunner, P. C. 2015. Emergence and early evolution of fungicide resistance in North American populations of Zymoseptoria tritici. Plant Pathol. 64:961-971.

Fan, J., Urban, M., Parker, J. E., Brewer, H. C., Kelly, S. L., HammondKosack, K. E., Fraaije, B. A., Liu, X., and Cools, H. J. 2013. Characterization of the sterol $14 \alpha$-demethylases of Fusarium graminearum identifies a novel genus-specific CYP51 function. New Phytol. 198:821-835.

Hall, T. A. 1999. BioEdit: A user-friendly biological sequence alignment editor and analysis program for Windows 95/98/NT. Nucleic Acids Symp. Ser. 41:95-98.

Hawkins, N. J., Cools, H. J., Sierotzki, H., Shaw, M. W., Knogge, W., Kelly, S. L., Kelly, D. E., and Fraaije, B. A. 2014. Paralog re-emergence: A novel, historically contingent mechanism in the evolution of antimicrobial resistance. Mol. Biol. Evol. 31:1793-1802.

Katoh, K., Asimenos, G., and Toh, H. 2009. Multiple alignment of DNA sequences with MAFFT. Methods Mol. Biol. 537:39-64.

Kendall, S. J., Hollomon, D. W., Cooke, L. R., and Jones, D. R. 1993. Changes in sensitivity to DMI fungicides in Rhynchosporium secalis. Crop Prot. 12: 357-362.
Lamari, L. 2002. ASSESS: Image Analysis Software for Plant Disease Quantification. The American Phytopathological Society, St. Paul, MN.

Librado, P., and Rozas, J. 2009. DnaSP v5: A software for comprehensive analysis of DNA polymorphism data. Bioinformatics 25:1451-1452.

Linde, C. C., Zala, M., and McDonald, B. A. 2009. Molecular evidence for recent founder populations and human mediated migration in the barley scald pathogen Rhynchosporium secalis. Mol. Phylogenet. Evol. 51:454464.

Liu, X., Yu, F., Schnabel, G., Wu, J., Wang, Z., and Ma, Z. 2011. Paralogous CYP51 genes in Fusarium graminearum mediate differential sensitivity to sterol demethylation inhibitors. Fungal Genet. Biol. 48:113-123.

McDonald, B. A. 2015. How can research on pathogen population biology suggest disease management strategies? The example of barley scald (Rhynchosporium commune). Plant Pathol. 64:1005-1013.

McDonald, B. A., Zhan, J., and Burdon, J. J. 1999. Genetic structure of Rhynchosporium secalis in Australia. Phytopathology 89:639-645.

Ohno, S. 1970. Evolution by Gene Duplication. Springer-Verlag, New York.

Simberloff, D. S. 1978. Use of rarefaction and related methods in ecology. Pages 150-165 in: Biological Data in Water Pollution Assessment: Quantitative and Statistical Analyses. K. L. Dickson, J. Cairns Jr., and R. J. Livingston, eds. Am. Soc. Test. Mater. 652, Philadelphia.

Stefansson, T. S., McDonald, B. A., and Willi, Y. 2013. Local adaptation and evolutionary potential along a temperature gradient in the fungal pathogen Rhynchosporium commune. Evol. Appl. 6:524-534.

Stefansson, T. S., McDonald, B. A., and Willi, Y. 2014. The influence of genetic drift and selection on quantitative traits in a plant pathogenic fungus. PLoS One 9:e112523.

Tajima, F. 1989. Statistical method for testing the neutral mutation hypothesis by DNA polymorphism. Genetics 123:585-595.

Tamura, K., Stecher, G., Daniel Peterson, D., Filipski, A., and Kumar, S. 2013. MEGA6: Molecular Evolutionary Genetics Analysis version 6.0. Mol. Biol. Evol. 30:2725-2729.

Untergasser, A., Cutcutache, I., Koressaar, T., Ye, J., Faircloth, B. C., Remm, M., and Rozen, S. G. 2012. Primer3-New capabilities and interfaces. Nucleic Acids Res. 40:e115.

Yan, X., Ma, W. B., Li, Y., Wang, H., Que, Y.-W., Ma, Z. H., Talbot, N. J., and Wang, Z. Y. 2011. A sterol 14[alpha]-demethylase is required for conidiation, virulence and for mediating sensitivity to sterol demethylation inhibitors by the rice blast fungus Magnaporthe oryzae. Fungal Genet. Biol. 48:144-153.

Yang, Z. 2007. PAML 4: Phylogenetic analysis by maximum likelihood. Mol. Biol. Evol. 24:1586-1591.

Zhan, J., Fitt, B. D., Pinnschmidt, H. O., Oxley, S. J. P., and Newton, A. C. 2008. Resistance, epidemiology and sustainable management of Rhynchosporium secalis populations on barley. Plant Pathol. 57:1-14.

Zhang, J. 2003. Evolution by gene duplication-an update. Trends Ecol. Evol. 18:292-298. 